\title{
INVARIANT DOMAINS FOR KLEINIAN GROUPS ${ }^{1}$
}

\author{
BY R. ACCOLA
}

Communicated by Lipman Bers, December 2, 1963

If the limit set, $\Sigma$, of a properly discontinuous group, $\Gamma$, of fractional linear transformations of the Riemann sphere, $S$, contains more than two points, call $\Gamma$ Kleinian. Otherwise, call $\Gamma$ elementary. Let $\left\{\Omega_{i}\right\}$ be an enumeration of the components of $\Omega$, the set of discontinuity. If $O$ is a domain in $S$, i.e., $O$ is open and connected, let $\Gamma(O)$ be the subgroup of $\Gamma$ of elements which map $O$ onto itself. If $\Gamma(O)=\Gamma$, call $O$ an invariant domain. If $\Gamma\left(\Omega_{i}\right)=\{i d\}$, call $\Omega_{i}$ an atom .

Theorem 1. If $\Gamma$ possesses three disjoint invariant domains then $\Gamma$ is cyclic.

TheOREM 2. Suppose $\Gamma$ possesses an invariant component $\Omega_{0}$. If $0 \neq i \neq j \neq 0$, then $\Gamma\left(\Omega_{i}\right) \cap \Gamma\left(\Omega_{j}\right)$ is a nonloxodromic and nonhyperbolic cyclic group. If $\Omega_{0}$ is simply connected this latter group is nonelliptic.

THEOREM 3. If $\Gamma$ is a Kleinian group with two disjoint invariant domains, then there exists a maximal pair of disjoint invariant domains ${ }^{2}$ each of which is simply connected. All noninvariant components of $\Omega$ are atoms.

The author is grateful to Leon Greenberg for pointing out how the next theorem follows from the methods used in proving the previous theorems and, essentially, from a deep theorem of Nielsen and Fenchel on Fuchsian groups.

TheOREM 4. If $O_{1}$ and $O_{2}$ are a maximal pair of disjoint invariant domains for a Kleinian group, $\Gamma$, then $O_{1} / \Gamma$ and $O_{2} / \Gamma$ are homeomorphic surfaces.

Examples are given where (a) $\Omega$ and $\Sigma$ are both connected and (b) where $\Gamma$ possesses two invariant components and atoms.

The proofs follow from remarks of which the following are typical. (1) A closed set, invariant under $\Gamma$, contains $\Sigma$. (2) The components of the complement of a closed connected set are simply connected. (3) If $O$ is a simply connected domain invariant under a loxodromic transformation, $T$, then there is a Jordan arc in $O$ invariant under $T$

1 Research supported by the Office of Naval Research.

2 Added in proof. $O_{1}$ and $O_{2}$ are a maximal pair of disjoint invariant domains if whenever $O_{1}^{\prime}$ and $O_{2}^{\prime}$ are a pair of disjoint invariant domains such that $O_{i} \subset O_{i}^{\prime}$, then $J_{i}=O_{i}^{\prime}$ for $i=1,2$. 
connecting the fixed points of $T$. (4) If $O_{1}$ and $O_{2}$ are disjoint simply connected domains invariant under a loxodromic $T$, the corresponding arcs, as in (3), divide $S$ into two Jordan regions, one or the other of which must contain any domain disjoint from $O_{1}$ and $O_{2}$. (5) If $O$ is a simply connected domain invariant under an elliptic $T$, then $O$ must contain a fixed point of $T$.

The examples are elaborations of the ideas in L. R. Ford, Automorphic functions, 2nd ed., Chelsea, 1951, pp. 55-59.

Brown UNIVERSITY

\section{DIFFERENTIABLE NORMS IN BANACH SPACES ${ }^{1}$}

BY GUILLERMO RESTREPO

Communicated by W. Rudin, January 20, 1964

1. Introduction. In $[4$, p. 28] S. Lang has asked whether or not a separable Banach space has an admissible norm of class $C^{1}$. In this note we indicate a proof of the following theorem, which characterizes those Banach spaces for which such a norm exists.

Theorem 1. A separable Banach space has an admissible norm of class $C^{1}$ if and only if its dual is separable.

It follows from this theorem that not even $C(I)$ possesses an admissible differentiable norm.

2. Preliminaries. Let $X$ be a Banach space with norm $\alpha$; we write $S_{\alpha}=\{x \mid \alpha(x)=1\}$ and $B_{\alpha}=\{x \mid \alpha(x) \leqq 1\}$. A norm in $X$ is admissible if it induces the same topology as does $\alpha$. The dual space is written $X^{*}$ and the norm dual to $\alpha$ is denoted by $\alpha^{*}$. An $f \in X^{*}$ is called a support functional to $B_{\alpha}$ at $x \in S_{\alpha}$ if $\alpha^{*}(f)=f \cdot x$; if $f$ has norm 1 , it is called a normalized support functional and is written $\nu_{x}$. A norm is smooth if there is a unique normalized support functional to $B_{\alpha}$ at each $x \in S_{\alpha}$. The norm $\alpha$ is differentiable at $x \neq 0$ if there is an $\alpha^{\prime}(x)$ $\in X^{*}$ such that

$$
\lim _{y \rightarrow x ; y \neq x} \frac{\left|\alpha(y)-\alpha(x)-\alpha^{\prime}(x) \cdot(y-x)\right|}{\alpha(y-x)}=0
$$

\footnotetext{
${ }^{1}$ Research partially supported by NSF Grant G-24471.
} 